Informationen und Kursunterlagen

biaggi \& partner, Kurssekretariat Egghölzliweg 4a, 3074 Muri

Tel. 0319517320

Fax 0319517210

E-Mail: jean.biaggi@bluewin.ch
Für den Nichtfinanzexperten sind Interpretation und Anwendung von finanzrelevanten Daten oft schwierig, zur effizienten Klinikführung sind sie heute jedoch unerlässlich. Die Einführung von DRG verunsichert und verwirrt zusätzlich; erste Erfahrungen zeigen, dass sie den Klinikbetrieb massgeblich beeinflussen.

Ziel dieses Kurses ist es, Kenntnisse der Grundlagen im Finanzwesen zu vermitteln, das Verständnis der Zusammenhänge im Finanzwesen zu fördern und erste Auswirkungen der DRG auf den Spitalalltag aufzuzeigen. Der Kurs soll Ärztinnen und Ärzten in leitender Stellung helfen, ihre Anliegen effizient vorzubringen und durchzusetzen. Sie werden dadurch ernstzunehmende Gesprächspartner auf Führungsebene. Grosser Raum wird der Diskussion mit praxiserfahrenen Experten sowie dem offenen Erfahrungsaustausch unter den Teilnehmenden beigemessen.

\section{Kursinhalt}

- Einführung in die Bilanz, Erfolgsrechnung und das betriebliche Rechnungswesen (Kostenstellenrechnung, Prozesskostenrechnung usw.);

- finanzielle Führung im Jahreszyklus von Planung, Budgetierung, Controlling und Reporting, Kennziffern als Mittel der Führungspraxis;
- DRG als Leistungsentschädigung;

- direkte Konsequenzen auf den Spitalbetrieb anhand erster Erfahrungen aus der Praxis;

- finanzgestützte Entscheide in besonderen Situationen.

Zielpublikum: Ärztinnen und Ärzte aller Fachgebiete in Kaderposition (Chefärzte/Leitende Ärzte/ Oberärzte).

\section{Dozenten}

- Dr. rer. pol. Heinz Locher, Unternehmensberater, Bern / Dr. rer. pol. Kurt Aeberhard, Innopool AG, Schüpfen;

- Dr. med. Markus Trutmann, DRG-Experte, Zürich/Dr. med. Gallus Burri, Chefarzt, Kantonsspital Uri.

Kursdaten: 6. und 7. November 2008

Kursort: Kongress-Hotel Ramada, Solothurn

Kurskosten: 2 Kurstage inklusive Mittagessen, Getränke, Pausenverpflegung, Infrastruktur und Kursunterlagen: Fr. 1900.-

Anmeldung: 30. August 2008

\title{
Führungsseminar für Oberärztinnen und Oberärzte
}

\section{Führungsprinzipien und Leadership}

\section{FMH-approved}

\author{
Informationen und Kurs- \\ unterlagen \\ biaggi \& partner, Kurssekretariat \\ Egghölzliweg 4a, 3074 Muri \\ Tel. 0319517320 \\ Fax 0319517210 \\ E-Mail: jean.biaggi@bluewin.ch
}

Führungsprinzipen kennen und Leadership entwickeln, das ist das Motto des Führungsseminars 2008/2009, das praxisbezogen auf wesentliche Fragen des Führungsalltags von Oberärztinnen und Oberärzten eingeht. Das Seminar gibt Oberärztinnen und Oberärzten die Möglichkeit, die wichtigsten Führungstools kennenzulernen, die eigenen Führungskompetenzen zu überdenken und diese auch strukturiert weiterzuentwickeln.

Ausgehend von der Analyse allgemeiner Führungsprinzipien (Führungsverständnis, Teamführung, Teamentwicklung, Konfliktmanagement, Kommunikation, Selbstmanagement, Umgang in komplexen Organisationen usw.) erhalten die Teilnehmenden Grundlagenkenntnisse und die Gelegenheit, eigene Ideen im Sinne des Leaderships zu entwickeln. Die im Kurs erworbenen Erkenntnisse und Erfahrungen sind ihnen darüber hinaus in ihrer weiteren beruflichen Laufbahn von Nutzen.

Das Seminar besteht aus vier Modulen, die thematisch aufeinander abgestimmt und als Einheit konzipiert sind. Es ist FMH-approved und wird von der SGC für die persönliche Fortbildung anerkannt. Der Kursbesuch wird offiziell attestiert.

Zielpublikum: Oberärztinnen und Oberärzte aller medizinischen Fachgebiete.

Dozenten: themenbezogen qualifizierte Fachexperten.

\section{Kursdaten}

- 30. und 31. Oktober 2008

- 27. und 28. November 2008

- 2. und 3. März 2009

- 27. und 28. April 2009

Kursort: Kongress und Kursaal Bern AG, Bern Kurskosten: 8 Kurstage inklusive Mittagessen, Getränke, Pausenverpflegung, Infrastruktur und Kursunterlagen: Fr. 4900.-

Anmeldung: 31. August 2008 